\title{
NEW APPROACH OF MHD BOUNDARY LAYER FLOW TOWARDS A POROUS STRETCHING SHEET VIA SYMMETRY ANALYSIS AND THE GENERALIZED EXP-FUNCTION METHOD
}

\author{
A.A. GABER ${ }^{1,2 *}$, M.H. SHEHATA ${ }^{1}$ \\ ${ }^{1}$ Department of Mathematics, College of Science, and Human Studies at Hotat Sudair, Majmaah \\ University, Saudi Arabia \\ ${ }^{2}$ Department of Mathematics, Faculty of Education, Ain Shams University, Roxy, Hiliopolis, Cairo, Egypt \\ *Corresponding author: aagaber6@gmail.com,a.gaber@mu.edu.sa
}

\begin{abstract}
Due to importance of the slip effect on modeling the boundary layer flows, symmetries and exact solution investigations have been introduced in this paper for studying the effect of a slip boundary layer on the stretching sheet through a porous medium. The exact solution of the investigating model is obtained in term of exponential via the generalized Exp-Function method. This solution satisfies the boundary conditions. Finally, the effect of parameters on the velocity field is studied.
\end{abstract}

\section{IntRoduction}

Symmetry group analysis based on the transformation groups, now known as Lie groups, is the most important solution method for the nonlinear problems in the literature. This approach is used to analysis the symmetries of the differential equations. Then, the corresponding symmetry groups can be used to simplify the analysis of the problems governing by the differential equations in the engineering science, mathematical physics, and mechanics. Lie groups characterize the symmetry of the differential equations and may be a point, a contact, and a potential or a nonlocal symmetry. It has also been verified that these kinds of groups can be represented by their infinitesimals that contain dependent variables, independent

Received March $8^{\text {th }}, 2020$; accepted May 12 $2^{\text {th }}, 2020$; published June $24^{\text {th }}, 2020$.

1991 Mathematics Subject Classification. 35Q35, 34B15.

Key words and phrases. boundary layer; exact solutions; generalized exp-function method.

(C)2020 Authors retain the copyrights of their papers, and all open access articles are distributed under the terms of the Creative Commons Attribution License. 
variables and the derivatives of dependent variables as arguments. In the last century, the application of the Lie groups has been developed by a number of mathematicians. Ovsiannikov [1], Olver [2], Ibragimov [3], and Bluman and Kumei [4] are some of the mathematicians who have huge number of studies in that field $[5-9]$.

The boundary layer [10-13] equations are especially interesting from a physical point of view because they have the capacity to admit a large number of invariant solutions i.e. basically closed-form solutions. In the present context, invariant solutions are meant to be a reduction to a simpler equation such as an ordinary differential equation (ODE). Prandtl's boundary layer equations admit more and different symmetry groups. Symmetry groups or simply symmetries are invariant transformations which do not alter the structural form of the equation under investigation (Bluman and Kumei [1]).

This work is organized as follows. The problem is formulated in Section 2 and in Section 3 we calculate the symmetries of the thermal boundary layer equations. All invariant solutions of the thermal boundary layer equations in Section 4. Finally, we show the effect of parameters on the velocity field.

\section{Formulation of the PROBlem}

We consider the steady state 2D magnetohydrodynamic (MHN) boundary layer, incompressible and viscous flow on stretching sheet through a porous medium, where $M$ is the magnetic parameter, $k_{p}$ is the permeability parameter and $f_{w}$ is the mass transfer parameter, which is positive for suction and negative for injection.

$$
\begin{aligned}
\frac{\partial u}{\partial x}+\frac{\partial v}{\partial y} & =0 \\
u \frac{\partial u}{\partial x}+v \frac{\partial u}{\partial y} & =v \frac{\partial^{2} u}{\partial y^{2}}-\frac{v}{k_{0}} u-\frac{\alpha_{0} B_{0}^{2}}{\rho} u
\end{aligned}
$$

In (1) $u$ and $v$ are the components of velocity respectively in the $\mathrm{x}$ and $\mathrm{y}$ directions, $k_{0}$ is the permeability of the porous medium, $B_{0}$ is magnetic field of uniform strength and $\sigma_{0}$ is electrical conductivity, $v=\frac{\mu}{\rho}$ is the kinematic viscosity, $\mu$ is the coefficient of fluid viscosity and $\rho$ is the fluid density. By using the boundary layer approximations and neglecting viscous dissipation.

The appropriate boundary conditions for the problem are given by

$$
\begin{aligned}
& u=B x, \quad v=v_{w} \text { at } \quad y=0, \\
& u \longrightarrow 0 \quad \text { at } \quad y=\infty
\end{aligned}
$$

where $\mathrm{B}$ is the stretching rate, $v_{w}$ is the wall velocity and the velocity components along $\mathrm{x}, \mathrm{y}$ coordinates, respectively, are

$$
u=\frac{\partial \psi}{\partial y} \quad \text { and } \quad v=\frac{-\partial \psi}{\partial x}
$$


where $\psi$ is the stream function.

Using the relations (3) in the boundary layer (2) and in the energy (1) we get the following equations

$$
\frac{\partial \psi}{\partial y} \frac{\partial^{2} \psi}{\partial y \partial x}-\frac{\partial \psi}{\partial x} \frac{\partial^{2} \psi}{\partial y^{2}}=v \frac{\partial^{3} \psi}{\partial y^{3}}-\frac{v}{k} \frac{\partial \psi}{\partial y}-\frac{\alpha_{0} B_{0}^{2}}{\rho} \frac{\partial \psi}{\partial y},
$$

The boundary conditions (2) then become

$$
\begin{aligned}
& \frac{\partial \psi}{\partial y}=B x, \quad \frac{\partial \psi}{\partial x}=v_{w} \quad \text { at } \quad y=0 \\
& \frac{\partial \psi}{\partial y} \longrightarrow 0 \quad \text { at } \quad y=\infty
\end{aligned}
$$

\section{Symmetry anAlysis FOR the BOUNDARY LAYER EQUATIONS}

Firstly, we shall derive the similarity solutions using the Lie-group method [11] under which (1) is invariant.

Consider the one-parameter $(\varepsilon)$ Lie group of infinitesimal transformations in $(x, y, \psi)$ given by Lie point symmetries

$$
\begin{aligned}
& x^{*}=x^{*}(x, t, \psi ; \varepsilon), \quad y^{*}=y^{*}(x, y, \psi ; \varepsilon) \\
& \psi^{*}=\psi^{*}(x, y, \psi ; \varepsilon) .
\end{aligned}
$$

With associated infinitesimal form

$$
\begin{aligned}
& x^{*}=x+\varepsilon \eta(x, y, \psi ; \varepsilon)+o\left(\varepsilon^{2}\right), \\
& y^{*}=y+\varepsilon \zeta(x, y, \psi ; \varepsilon)+o\left(\varepsilon^{2}\right), \\
& \psi^{*}=\psi+\varepsilon \psi(x, y, \psi ; \varepsilon)+o\left(\varepsilon^{2}\right),
\end{aligned}
$$

where " $\varepsilon$ " is a small parameter.

If we set:

$$
\Delta_{1}=\frac{\partial \psi}{\partial y} \frac{\partial^{2} \psi}{\partial y \partial x}-\frac{\partial \psi}{\partial x} \frac{\partial^{2} \psi}{\partial y^{2}}-v \frac{\partial^{3} \psi}{\partial y^{3}}+\left(\frac{v}{k}+\frac{\alpha_{0} B_{0}^{2}}{\rho}\right) \frac{\partial \psi}{\partial y},
$$

The invariance conditions[1-4]

$$
\Gamma^{(3)}\left(\Delta_{\alpha}\right)=0 \quad \text { whenever } \quad \Delta_{\alpha}=0, \alpha=1,2,
$$

where $\Gamma^{(3)}$ is given by

$$
\Gamma^{(3)}=\chi+g^{x} \frac{\partial}{\partial \psi_{x}}+g^{x x} \frac{\partial}{\partial \psi_{x x}}+g^{x t} \frac{\partial}{\partial \psi_{x t}}+g^{x x x} \frac{\partial}{\partial \psi_{x x x}} .
$$

where

$$
\chi=\zeta \frac{\partial}{\partial x}+\tau \frac{\partial}{\partial y}+g \frac{\partial}{\partial \psi}
$$


The components $\zeta^{x}, \zeta^{y}, \tau^{x}, \tau^{y}, g^{x}, g^{x x}, g^{x x y}$...can be determined from the following expressions:

$$
g^{s}=D_{s} g-\psi_{t} D_{s} \zeta-\psi_{x} D_{s} \tau, \quad g^{s j}=D_{j} g-\psi_{t s} D_{j} \zeta-\psi_{x s} D_{j} \tau
$$

Equation (9) gives the following system of linear partial differential equations:

$$
\begin{gathered}
\zeta_{y}=0, \quad \zeta_{\psi}=0, \\
g_{y}=0, \quad g_{x}=0, \\
\tau_{y}=0, \quad \tau_{\psi}=0, \\
g_{\psi \psi}=0, \quad \zeta_{x}-g_{\psi}=0,
\end{gathered}
$$

Solving the system (13), after substitution from (12) into(13), and using the invariance of the boundary conditions (6), yields

$$
\begin{aligned}
& \zeta=\lambda_{1} x+\lambda_{2} \\
& \tau=\lambda_{3}(x) \\
& g=\lambda_{1} \psi+\lambda_{4}
\end{aligned}
$$

In order to study the group theoretic structure, the vector field operator $\mathrm{V}$ is written as

$$
V=V_{1}\left(\lambda_{1}\right)+V_{2}\left(\lambda_{2}\right)+V_{3}\left(\lambda_{3}\right)+V_{4}\left(\lambda_{4}\right)
$$

where

$$
\begin{aligned}
& V_{1}=x \frac{\partial}{\partial x}+\psi \frac{\partial}{\partial \psi}, V_{2}=\frac{\partial}{\partial x} \\
& V_{3}=\lambda_{3}(x) \frac{\partial}{\partial y}, \quad V_{4}=\frac{\partial}{\partial \psi} .
\end{aligned}
$$

It is easy to verify, that the vector fields are closed under the Lie bracket as follows

$\left[V_{1}, V_{1}\right]=\left[V_{2}, V_{2}\right]=\left[V_{3}, V_{3}\right]=\left[V_{4}, V_{4}\right]=\left[V_{1}, V_{3}\right]=0$

$\left[V_{2}, V_{3}\right]=\left[V_{2}, V_{4}\right]=0,\left[V_{3}, V_{1}\right]=\left[V_{3}, V_{2}\right]=\left[V_{3}, V_{4}\right]=0$

$\left[V_{4}, V_{2}\right]=\left[V_{4}, V_{3}\right]=0,\left[V_{1}, V_{2}\right]=-\left[V_{2}, V_{1}\right]=-V_{2}$

$\left[V_{1}, V_{4}\right]=-\left[V_{4}, V_{1}\right]=-V_{4}$

Further, from the symmetries given in (16) the following possibilities exist for the solution of (9).

(I) $V_{1}$

(II) $V_{2}+V_{3}$

(III) $V_{2}+V_{3}+V_{4}$ 
Having determined the infinitessimals, the symmetry variables are found by solving the auxiliary equation

$$
\frac{d x}{\zeta}=\frac{d y}{\tau}=\frac{d \psi}{g} .
$$

\section{Reductions and exact solutions}

Now we look the similarity solutions with respect to the generators $V_{1}$

$$
\eta^{*}=y, \quad \psi=x F\left(\eta^{*}\right)
$$

The reduced system of ODEs is

$$
F^{22}-F F^{\prime \prime}-v F^{\prime \prime \prime}+\left(\frac{v}{k}+\frac{\alpha_{0} B_{0}^{2}}{\rho}\right) F^{\prime}=0,
$$

The boundary condition take the following forms

$$
\begin{array}{ll}
F^{\prime}=B, \quad F=v_{w} & \text { at } \quad \eta^{*}=0 \\
F^{\prime}=0 & \text { at } \quad \eta^{*} \rightarrow \infty .
\end{array}
$$

We look for a similarity solution of (19) , and boundary condition (20) as the following form:

$$
F=\sqrt{B v} f(\eta) \text { and } \eta=\sqrt{\frac{B}{v}} \eta^{*}
$$

Using (21) we obtain the following self-similar equations

$$
f^{\prime \prime \prime}-f^{\prime 2}+f f^{\prime \prime}-\left(k_{p}+M\right) f^{\prime}=0,
$$

subject to the boundary conditions

$$
f(0)=f_{w}, \quad f^{\prime}(0)=1 \quad f^{\prime}(\infty)=0
$$

where $M=\frac{\alpha_{0} B_{0}^{2}}{\rho B}$ is the magnetic field, $k_{p}=\frac{v}{k_{0} B}$ is the permeability of the porous medium and $f_{w}=\frac{v_{w}}{\sqrt{B v}}$ where $f_{w}>0$ corresponds to suction and $f_{w}<0$ for injection. Equation (22) is nonlinear differential equation which can be solved by the generalized He's Exp-Function method. In view of the generalized Exp-Function method [14-16], we assume that the solution of (22) can be expressed in the form

$$
f(\eta)=\frac{a_{-c}\left[\phi(\eta)^{-c}\right]+\ldots+a_{p}\left[\phi(\eta)^{p}\right]}{r_{-d}\left[\phi(\eta)^{-d}\right]+\ldots+r_{q}\left[\phi(\tau)^{q}\right]}
$$

where $c, d, p$ and $q$ are positive integers which are unknown to be further determined, $a_{n}$ and $r_{m}$ are unknown constants. In addition, $\phi(\eta)$ satisfies Riccati equation,

$$
\phi^{\prime}(\eta)=A+B \phi(\eta)+C \phi(\eta)^{2} .
$$


In order to determine values of $c$ and $p$, we balance the linear term of the highest order in Eq. (24) with the highest order nonlinear term $f^{\prime \prime \prime}$ and $f^{\prime 2}$, we have

$$
\begin{gathered}
f^{\prime \prime \prime}(\eta)=\frac{a_{1} \phi^{-c-8 d-3}+\ldots+a_{2} \phi^{p+8 q+3}}{r_{1} \phi^{-9 d}+\ldots+r_{2} \phi^{9 q}}, \\
f^{\prime 2}(\eta)=\frac{a_{3} \phi^{-2 c-6 d-2}+\ldots+a_{4} \phi^{2 p+6 q+2}}{r_{3} \phi^{-9 d}+\ldots+r_{4} \phi^{9 q}},
\end{gathered}
$$

where $a_{i}$ and $r_{i}$ are determined coefficients only for simplicity. From balancing the lowest order and highest order of $\phi(26)$ and (27), we obtain $-7 d-c-3=-6 d-2 c-2$, which leads to the limit $c=d+1$,and $7 q+p+3=6 q+2 p+2$, which leads to the limit $p=q+1$, for simplicity $d=q=0$, the function in Eq. (24), becomes

$$
f(\eta)=\gamma_{-1} \phi^{-1}+\gamma_{0}+\gamma_{1} \phi
$$

Substituting (28) into (22), equating to zero the coefficients of all powers of $\phi(\eta)$ yields a set of algebraic equations for $\gamma_{0}, \gamma_{1}$ and $\gamma_{-1}$, we obtain the following system

$$
\begin{aligned}
& \gamma_{1}^{2} B C+12 \gamma_{1} B C^{2}+2 \gamma_{0} \gamma_{1} C^{2}=0 \\
& -6 \gamma_{-1} A^{3}+\gamma_{-1}^{2} A^{2}, \gamma_{1}^{2} C^{2}+6 \gamma_{1} C^{3}=0 \\
& -12 \gamma_{-1} A^{2} B+\gamma_{-1}^{2} A B+2 \gamma_{0} \gamma_{-1} A^{2}=0 \\
& -6 \gamma_{-1} A^{3}+\gamma_{-1}^{2} A^{2}=0 \\
& \gamma_{1}^{2} B C+12 \gamma_{1} B C^{2}+2 \gamma_{0} \gamma_{1} C^{2}=0 \\
& -12 \gamma_{-1} A^{2} B+\gamma_{-1}^{2} A B+2 \gamma_{0} \gamma_{-1} A^{2}-M \gamma_{1} B+5 \gamma_{-1} \gamma_{1} B C-\left(2\left(-\gamma_{-1} C+\gamma_{1} A\right)\right) \gamma_{1} B \\
& +\gamma_{1}\left(8 A B C+B^{3}\right)+\gamma_{1}\left(\gamma_{-1} B C+\gamma_{1} A B\right)+\gamma_{0} \gamma_{1}\left(2 A C+B^{2}\right)=0 \\
& -\left(2\left(-\gamma_{-1} C+\gamma_{1} A\right)\right) \gamma_{1} C-\gamma_{1}^{2} B^{2}+2 \gamma_{-1} \gamma_{1} C^{2}+3 \gamma_{0} \gamma_{1} B C+\gamma_{1}^{2}(2 A C+B 2)+\gamma_{1}\left(8 A C^{2}+7 B^{2} C\right) \\
& -M \gamma_{1} C, 5 \gamma_{1} \gamma_{-1} A B+2 \gamma_{-1} B\left(-\gamma_{-1} C+\gamma_{1} A\right)+M \gamma_{-1} B-\gamma_{-1}\left(8 A B C+B^{3}\right)+\gamma_{0} \gamma_{-1}\left(2 A C+B^{2}\right) \\
& +\gamma_{-1}\left(\gamma_{-1} B C+\gamma_{1} A B\right)+24 \gamma_{-1} A B C+6 \gamma_{-1} B\left(2 A C+B^{2}\right)-6 \gamma_{-1}\left(4 A B C+B\left(2 A C+B^{2}\right)\right)=0
\end{aligned}
$$

Solving the system of algebraic equations with the aid of Maple, we obtain the following results:

$$
\begin{aligned}
& \gamma_{-1}=\frac{\left(\left(k_{p}+M\right)-B^{2}+\gamma_{0} B\right)}{C}, \gamma_{1}=0 \text { at } A=0 . \\
& \gamma_{-1}=-6 C, \quad \gamma_{1}=6 A \text { at } B=0
\end{aligned}
$$


Substituting (30) into (28), the solutions of (1) can be written as:

$$
f(\eta)=\gamma_{0}-\frac{\left(\left(k_{p}+M\right)-B^{2}+\gamma_{0} B\right)}{C} \frac{C \exp (B \eta)-1}{B \exp (B \eta)}
$$

where $\gamma=\frac{a_{-1}}{r_{-1}}$. Now we have to apply the boundary conditions to the solution (19), noting that the third one is already satisfied. On using the first two boundary conditions we then need to solve the system:

$$
\begin{aligned}
\gamma_{0}-\frac{\left(\left(k_{p}+M\right)-B^{2}+\gamma_{0} B\right)}{C} \frac{C-1}{B} & =f_{w}, \\
-B \gamma+B^{2}-M+\frac{\left(\left(k_{p}+M\right)-B^{2}+\gamma_{0} B\right)(C-1)}{C} & =1 .
\end{aligned}
$$

By solving Eq. (33) then substituting in Eq. (32), we obtain the closed form solution

$$
f(\eta)=\gamma_{0}-\frac{\left(\left(k_{p}+M\right)-B^{2}+\gamma_{0} B\right)}{B}+\frac{\left(\left(k_{p}+M\right)-B^{2}+\gamma_{0} B\right)}{C B \exp (B \eta)},
$$

where $C \neq 0, \gamma_{0}=\frac{-C+1+f_{w}\left(\frac{1}{2} f_{w}+\frac{1}{2} \sqrt{f_{w}^{2}+4+4\left(k_{p}+M\right)}\right)}{\frac{1}{2} f_{w}+\frac{1}{2} \sqrt{f_{w}^{2}+4+4\left(k_{p}+M\right)}}$ and $B=\frac{1}{2} f_{w}+\frac{1}{2} \sqrt{f_{w}^{2}+4+4\left(k_{p}+M\right)}$.

\section{Results And Discussion}

Figs. 1-3 have been made in order to see the effects of the permeability of the porous medium $k_{p}$, suction/injection parameter $f_{w}$ and the MHD parameter M on the velocity field.

Fig. (1) From this figure, rise in $M$ indicates the raise of magnetic field which acts like a resistive force and consequently fluid flow slowdowns relatively and hence boundary layer thickness increases.

Fig. (2) The effect of the influence of the porous medium on horizontal velocity. It is found that the horizontal velocity decreases with the increase of $\mathrm{k}$ i.e. Increased permeability parameter $\left(k_{p}\right)$ caues an increase in resistance to fluid along the surface, and this leads to increase the thickness of the boundary layer.

Fig. (3) show the effects of suction $\left(f_{w}>0\right)$ and injection $\left(f_{w}<0\right)$ on the horizontal velocity $f^{\prime}(\eta)$ the effect of suction is to decrease the horizontal velocity whereas the effect of injection is to increase this.

\section{Conclusion}

In this paper, the couple system of MHD boundary layer flow towards a porous stretching sheet have been reduced by symmetry method to ordinary differential equations. the exact solutions of ordinary differential equations is obtained by the generalized Exp-Function method. Finally, some plots have been given for study the effects of various parameters on velocity of fluid .

\section{Acknowledgements}

The authors would like to thank the deanship of scientific research of Majmaah niversity for the financial grant received for conducting this research. 


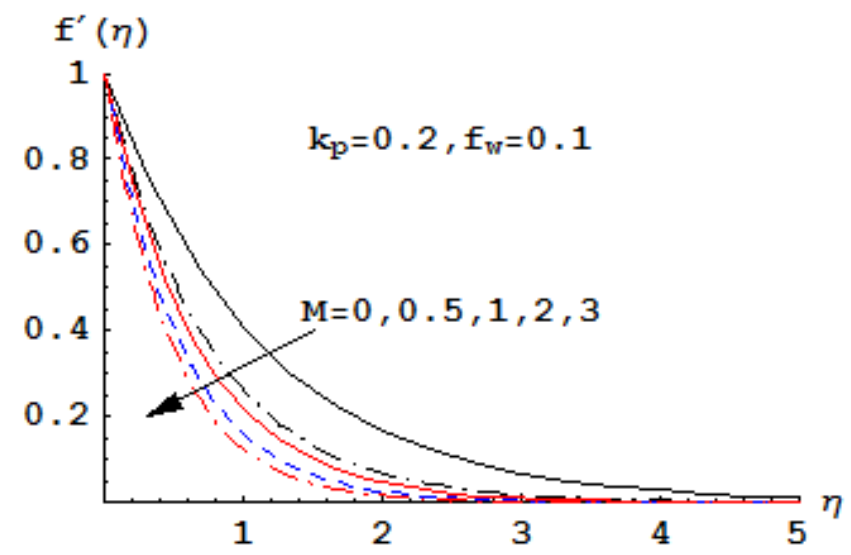

FiguRE 1. Velocity profile for different values of magnatic field M

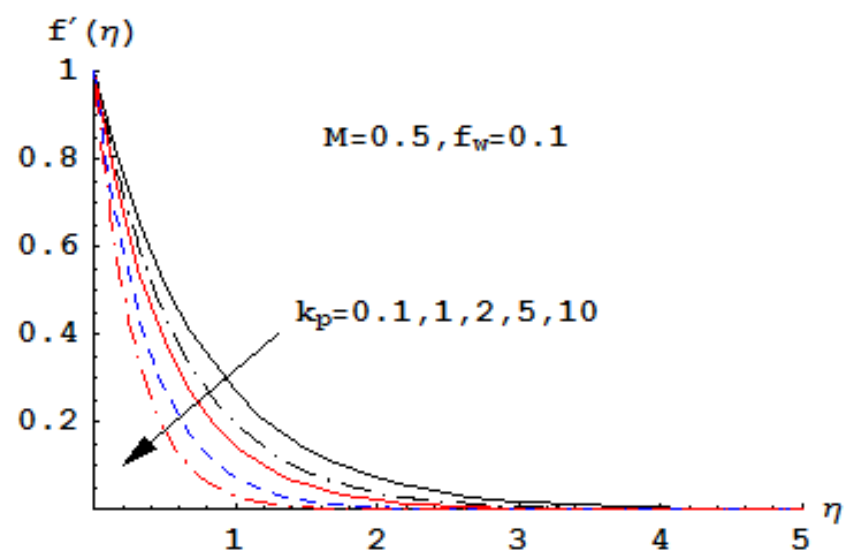

FigURE 2. Velocity profile for different values of porous medium $K_{p}$

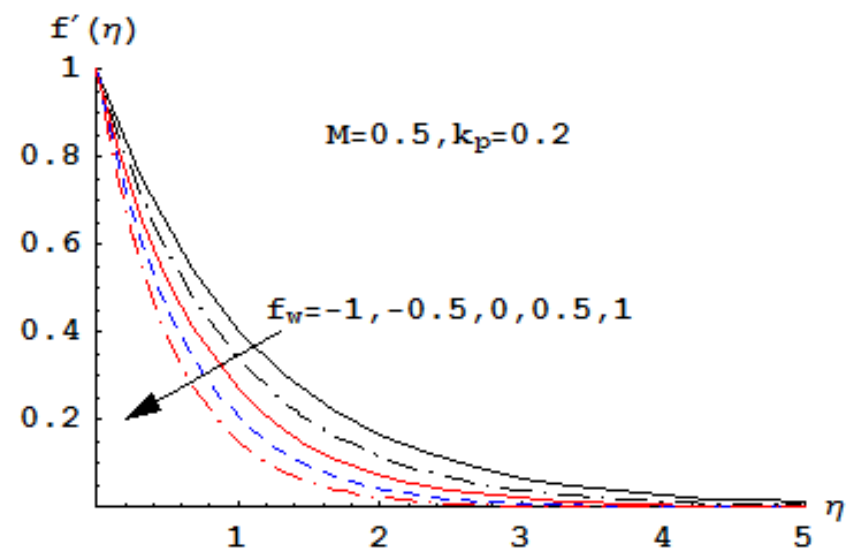

Figure 3. Velocity profile for several values of $f_{w}$ 
APPENDIX A: The exact solutions for Riccati Equation.

\begin{tabular}{lllll}
\hline cases & $\mathrm{A}$ & $\mathrm{B}$ & $\mathrm{C}$ & solutions of Ricati equation \\
\hline & & & & \\
1 & $\neq 0$ & $\neq 0$ & $\neq 0$ & $\frac{-B+\sqrt{4 A C-B^{2}} \tan \left(\frac{1}{2}\left(\sqrt{4 A C-B^{2}}\left(\zeta+d_{0}\right)\right)\right.}{2 C}$ \\
2 & free & $\neq 0$ & 0 & $\frac{-A}{B}+\exp (B \zeta)$ \\
3 & 0 & $\neq 0$ & $\neq 0$ & $\frac{-B \exp \left(B \zeta+B d_{0}\right)}{C \exp \left(B \zeta+B d_{0}\right)-1}$ \\
4 & 0 & -1 & -1 & $\frac{-d_{0}}{\exp (\zeta)+d_{0}}$ \\
\hline
\end{tabular}

Conflicts of Interest: The author(s) declare that there are no conflicts of interest regarding the publication of this paper.

\section{REFERENCES}

[1] L.V. Ovsiannikov, Group analysis of differential equations. Nauka, Moscow, 1978.

[2] P.J. Olver, Application of lie groups to differential equations. Springer-Verlag, 1986.

[3] N.K. Ibragimov, CRC handbook of Lie group analysis of differential equations, CRC Press, Boca Raton, 1994.

[4] G.W. Bluman, S. Kumei, Symmetries and Differential Equations, Springer-Verlag, New York, 1989.

[5] M.F. El-Sayed, G.M. Moatimid, M.H.M. Moussa, R.M. El-Shiekh, F.A.H. El-Shiekh, A.A. El-Satar, A study of integrability and symmetry for the $(\mathrm{p}+1)$ th Boltzmann equation via Painlevé analysis and Lie-group method, Math. Meth. Appl. Sci. 38 (2015), 3670-3677.

[6] M.H.M. Moussa, A.A. Gaber, Symmetry Analysis and Solitary Wave Solutions of Nonlinear Ion-Acoustic Waves Equation, Int. J. Anal. Appl. 18 (3) (2020), 448-460.

[7] M. Rosa, J.C. Camacho, M.S. Bruzón, M.L. Gandarias, Classical and potential symmetries for a generalized Fisher equation, J. Comput. Appl. Math. 318 (2017), 181-188.

[8] J.C. Camacho, M. Rosa, M.L. Gandarias, M.S. Bruzón, Classical symmetries, travelling wave solutions and conservation laws of a generalized Fornberg-Whitham equation, J. Comput. Appl. Math. 318 (2017), 149-155.

[9] R. Sinuvasan, K.M. Tamizhmani, P.G.L. Leach, Symmetries, travelling-wave and self-similar solutions of the Burgers hierarchy, Appl. Math. Comput. 303 (2017), 165-170.

[10] M. F. El-Sayed, G. M. Moatimid, M. H. M. Moussa, R. M. El-Shiekh and A. A. El-Satar, Similarity Reductions and New Exact Solutions for B-Family Equations, Amer. J. Math. Stat. 2 (3) (2012), 40-43

[11] B.C. Sakiadis, Boundary-layer behavior on continuous solid surfaces: I. Boundary-layer equations for two-dimensional and axisymmetric flow, AIChE J. 7 (1961), 26-28.

[12] M. Hatami, D.D. Ganji, Heat transfer and nanofluid flow in suction and blowing process between parallel disks in presence of variable magnetic field, J. Mol. Liquids. 190 (2014), 159-168.

[13] K. Bhattacharyya, S. Mukhopadhyay, G.C. Layek, I. Pop, Effects of thermal radiation on micropolar fluid flow and heat transfer over a porous shrinking sheet, Int. J. Heat Mass Transfer. 55 (2012), 2945-2952.

[14] A. Ebaid and Abdul-Majid Wazwaz, On the Generalized Exp-Function Method and Its Application to Boundary Layer Flow at Nano-Scale J. comput. theor. nanoscience, (2014), 11, 1-7

[15] A.E.-H. Ebaid, Generalization of He's Exp-Function Method and New Exact Solutions for Burgers Equation, Z. Naturforsch., A. 64 (2009), 604-608. 
[16] E.M.E. Zayed, A.-G. Al-Nowehy, Exact solutions and optical soliton solutions for the $(2+1)$-dimensional hyperbolic nonlinear Schrödinger equation, Optik. 127 (2016), 4970-4983. 